\title{
TNM staging with FDG-PET/CT in patients with primary head and neck cancer
}

Veit-Haibach, Patrick ; Luczak, Christopher ; Wanke, Isabel ; Fischer, Markus ; Egelhof, Thomas ; Beyer, Thomas ; Dahmen, Gerlinde ; Bockisch, Andreas ; Rosenbaum, Sandra ; Antoch, Gerald

\begin{abstract}
Purpose: PET/CT, PET+CT, and CT were compared concerning accuracies in TNM staging and malignancy detection in head and neck cancer. The impact of PET/CT compared to the other imaging modalities on therapy management was assessed. Materials and methods: Fifty-five patients with suspected head and neck primary cancer underwent whole-body FDG-PET/CT. PET/CT and PET+CT were evaluated by a nuclear medicine physician and a radiologist; CT was evaluated by two radiologists, PET by two nuclear physicians. Histopathology served as the standard of reference. Differences between the staging modalities were tested for statistical significance by McNemar's test. Results: Overall TNMstaging and T-staging with PET/CT were more accurate than PET+CT and CT alone $(\mathrm{p}<0.05)$. $\mathrm{PET} / \mathrm{CT}$ was marginally more accurate than $\mathrm{CT}$ alone in $\mathrm{N}$-staging $(\mathrm{p}=0.04)$; no statistically significant difference was found when compared to PET $+\mathrm{CT}$ for N-staging. PET/CT altered further treatment in 13 patients compared to CT only and in 7 patients compared to PET+CT. Conclusion: Combined PET/CT proved to be partly more accurate in assessing the overall TNM-stage than CT and PET+CT. These results were based on a higher accuracy concerning the T-stage, mainly in patients with metallic implants and marginally the N-stage. Therapy decisions have been influenced in a substantial number of patients. PET/CT might be considered as a first line diagnostic tool in patients with suspected primary head and neck cancer
\end{abstract}

DOI: https://doi.org/10.1007/s00259-007-0564-5

Posted at the Zurich Open Repository and Archive, University of Zurich

ZORA URL: https://doi.org/10.5167/uzh-156109

Journal Article

Published Version

Originally published at:

Veit-Haibach, Patrick; Luczak, Christopher; Wanke, Isabel; Fischer, Markus; Egelhof, Thomas; Beyer, Thomas; Dahmen, Gerlinde; Bockisch, Andreas; Rosenbaum, Sandra; Antoch, Gerald (2007). TNM staging with FDG-PET/CT in patients with primary head and neck cancer. European Journal of Nuclear Medicine and Molecular Imaging, 34(12):1953-1962.

DOI: https://doi.org/10.1007/s00259-007-0564-5 


\title{
TNM staging with FDG-PET/CT in patients with primary head and neck cancer
}

\author{
Patrick Veit-Haibach • Christopher Luczak • \\ Isabel Wanke • Markus Fischer • Thomas Egelhof • \\ Thomas Beyer • Gerlinde Dahmen • Andreas Bockisch • \\ Sandra Rosenbaum • Gerald Antoch
}

Received: 21 May 2007 / Accepted: 25 July 2007 / Published online: 24 August 2007

(C) Springer-Verlag 2007

\begin{abstract}
Purpose $\mathrm{PET} / \mathrm{CT}$, PET $+\mathrm{CT}$, and $\mathrm{CT}$ were compared concerning accuracies in TNM staging and malignancy detection in head and neck cancer. The impact of PET/CT
\end{abstract}

P. Veit-Haibach $(\bowtie) \cdot$ C. Luczak $\cdot$ I. Wanke $\cdot$ T. Egelhof $\cdot$

G. Antoch

Department of Diagnostic and Interventional Radiology

and Neuroradiology, University Hospital Essen,

Hufelandstrasse 55,

45122 Essen, Germany

e-mail: patrick.veit-haibach@usz.ch

\section{Fischer}

Department of Oto-Rhino-Laryngology, University Hospital Essen,

Essen, Germany

T. Beyer $\cdot$ A. Bockisch $\cdot$ S. Rosenbaum

Department of Nuclear Medicine, University Hospital Essen,

Essen, Germany

G. Dahmen

Institute of Medical Biometry and Statistics, University at Luebeck,

Luebeck, Germany

Present address:

P. Veit-Haibach

Department for Nuclear Medicine, University Hospital Zuerich,

Raemistrasse 100,

8091 Zuerich, Switzerland

Present address:

M. Fischer

HNO-Facharztpraxis,

Kuno-Fischer-Strasse 4,

69120 Heidelberg, Germany

Present address:

T. Egelhof

Dept. of Radiology, University Hospital Basel,

Hebelstrasse 32,

9031 Basel, Switzerland compared to the other imaging modalities on therapy management was assessed.

Materials and methods Fifty-five patients with suspected head and neck primary cancer underwent whole-body FDG-PET/CT. PET/CT and PET+CT were evaluated by a nuclear medicine physician and a radiologist; CT was evaluated by two radiologists, PET by two nuclear physicians. Histopathology served as the standard of reference. Differences between the staging modalities were tested for statistical significance by McNemar's test.

Results Overall TNM-staging and T-staging with PET/CT were more accurate than PET $+\mathrm{CT}$ and $\mathrm{CT}$ alone $(\mathrm{p}<0.05)$. $\mathrm{PET} / \mathrm{CT}$ was marginally more accurate than $\mathrm{CT}$ alone in $\mathrm{N}$ staging $(\mathrm{p}=0.04)$; no statistically significant difference was found when compared to PET+CT for $\mathrm{N}$-staging. PET/CT altered further treatment in 13 patients compared to $\mathrm{CT}$ only and in 7 patients compared to $\mathrm{PET}+\mathrm{CT}$.

Conclusion Combined PET/CT proved to be partly more accurate in assessing the overall TNM-stage than CT and $\mathrm{PET}+\mathrm{CT}$. These results were based on a higher accuracy concerning the T-stage, mainly in patients with metallic implants and marginally the N-stage. Therapy decisions have been influenced in a substantial number of patients. PET/CT might be considered as a first line diagnostic tool in patients with suspected primary head and neck cancer.

Keywords Contrast-enhanced PET/CT · TNM staging · Head and neck cancer. Therapeutic influence

\section{Introduction}

Head and neck cancer is one of the top ten leading cancers as regard incidence [1]. Therapy of head and neck cancer patients is complex, but multiple therapeutic regimens have been developed over the past few years. Head and neck cancer 
therapy requires a multi-disciplinary approach including oncology, surgery, radiotherapy and imaging service [2]. Initial staging is of particular importantce, since recurrent and residual disease in head and neck cancer frequently do not respond sufficiently to available therapy options [3-5]. Detection of head and neck tumours is performed clinically with endoscopic procedures; however, tumour size, depth of infiltration and the nodal status often evade characterisation.

Based on international staging guidelines morphology imaging of the head and neck by means of CT and/or MRI represents the method-of-choice for staging head and neck cancer today $[2,6,7]$. Nevertheless, anatomical imaging options are limited with respect to the detection of the tumour sites and evaluation of the nodal disease [8-10]. Furthermore, evaluation of morphological imaging can be challenging based on the complex anatomy of the head and neck area. In comparison, $\left({ }^{18} \mathrm{~F}\right)$-fluoro-2-deoxy-D-glucose (FDG) positron emission tomography (PET) has shown very good results concerning tumour detection and detection of nodal metastases $[8,11,12]$. Sensitivities and specificities for FDG-PET in head and neck cancer are as high as $87 \%$ and $94 \%$, compared to as low as $67 \%$ and $25 \%$ for CT and MRI, respectively [11, 12]. Recently, the combination of morphology and function within a single, integrated PET/CT tomograph demonstrated promising results concerning the detection and characterisation of head and neck lesions. Furthermore, a potentially positive impact on patient management has been indicated [13-16]. However, most of the available head and neck cancer studies today address the accuracy of PET/CT for lesion detection rather than for complete TNM staging. Furthermore, the majority of FDG-PET/CT scans included in these studies are performed without CT contrast enhancement, thus, rendering the $\mathrm{CT}$ images of poorer quality compared to standard radiology examinations.

This study aims at assessing the diagnostic accuracy for TNM staging in patients with suspected head and neck cancer. First, the diagnostic accuracies for TNM staging with combined $\mathrm{PET} / \mathrm{CT}$, CT-only and PET $+\mathrm{CT}$ (viewed side by side) were compared. Second, we evaluated the lesion detection rates (sensitivity, specificity, accuracy, NPV and PPV) for the primary head and neck tumour sites, nodal metastases and distant metastases for all four imaging modalities (CT-only, PET-only, PET + CT side by side and combined PET/CT). Finally, we assessed the impact of PET/CT on patient management compared to the $\mathrm{PET}+\mathrm{CT}$ and CT-only.

\section{Patients and methods}

\section{Patients}

The study was performed in accordance with the regulations of the local institutional review board and ethics committee for retrospective analysis. Informed consent was obtained from all patients prior to the examination. Fifty-five patients (mean age: 58 years; range: $34-94$ years, 7 female, 48 male) were included. All patients were referred for a combined FDG-PET/CT examination based on the clinical suspicion of a primary head and neck cancer. None of the patients suffered from a known inflammatory disease in the head and neck area. Local biopsy and histopathological workup was performed in all patients after the FDG-PET/CT scan.

\section{FDG-PET/CT imaging procedure}

PET/CT imaging was conducted on a Biograph Duo PET/CT system (Siemens Molecular Imaging, Hoffman Estates, IL). The system is composed of a dual-slice CT scanner (Somatom Emotion, Siemens Medical Solutions, Forchheim, Germany) and a full-ring, BGO-based PET tomograph (Siemens Molecular Imaging, Hoffman Estates, IL). The axial field-ofview and in-plane, spatial resolution of the PET is $15.5 \mathrm{~cm}$ per bed position and $4.6 \mathrm{~mm}$, respectively. Glucose levels in all patients were measured prior to the FDG injection. The average administered FDG dose was $340 \mathrm{MBq} 60 \mathrm{~min}$ prior to the PET/CT examination. During the uptake time $1,500 \mathrm{ml}$ of a water-based, negative oral contrast agent was applied for small bowel distension [17, 18].

A split imaging protocol was used for staging purposes. For the first part of the scan patients were positioned supine with their arms raised above the head.Following the CT topogram (scout scan) a spiral CT scan was performed in the caudo-cranial direction covering an axial imaging range from the apex of the lungs to the mid-thighs. CT images were acquired with $110 \mathrm{mAs}, 120 \mathrm{kVp}, 5$-mm slice thickness, and a 2.4-mm incremental reconstruction. Contrast enhancement was applied by means of $90 \mathrm{ml}$ of an iodinated contrast agent (300 $\mathrm{mmol} / \mathrm{ml}$, Guerbet GmbH, Sulzbach, Germany) with a 50 -s delay in starting the $\mathrm{CT}$ and a flow rate of $3 \mathrm{ml} / \mathrm{s}$. Patients were instructed to hold their breath in normal expiration for the duration of the CT scan of the lower thorax [19]. Subsequently, emission data were acquired for the same co-axial field-of-view. The average PET acquisition time per bed position was 3-5 min, depending on the weight of the patient. PET images were corrected for scatter and attenuation based on the available CT transmission images.

Following the torso scan patients were repositioned with their arms down and the head and neck placed in a VacLock bag for the second part of the scan [20]. Following the CT topogram scan a spiral CT scan was performed covering and axial imaging range from the upper skull to the apical lobes of the lung. A start delay of $30 \mathrm{~s}$ was chosen for the second contrast injection $(70 \mathrm{ml}$ of $300 \mathrm{mmol} / \mathrm{ml}$, Guerbet $\mathrm{GmbH}$, Sulzbach, Germany, flow rate: $3 \mathrm{ml} / \mathrm{s}$ ). CT images in the head and neck area were acquired with $110 \mathrm{mAs}$, $120 \mathrm{kVp}, 3-\mathrm{mm}$ slice thickness and a 2.4-mm incremental 
reconstruction. Corresponding PET acquisition time was $3.5 \mathrm{~min}$ for each of the two bed positions covering the same co-axial imaging range as the CT. Following CT-based scatter and attenuation correction PET images were reconstructed on $256 \times 256$ matrices, four iterations and eight subsets to enhance visibility of smaller structures. Corrected PET images were reconstructed iteratively (FORE-OSEM).

$\mathrm{CT}$ as well as PET data sets were viewed separately (CT, PET, PET $+\mathrm{CT})$ or in fused mode $(\mathrm{PET} / \mathrm{CT})$ on a commercially available computer workstation (Siemens Molecular Imaging, Erlangen, Germany). Thus, all compared imaging modalities derived from the same data set.

Image evaluation

\section{TNM staging}

For this study, TNM staging was performed exclusively for CT-only, PET $+\mathrm{CT}$ and PET/CT. The PET images were evaluated with and without attenuation correction by two nuclear medicine specialists in consensus. The CT images were evaluated by two radiologists in consensus. Fused PET/ $\mathrm{CT}$ and $\mathrm{PET}+\mathrm{CT}$ viewed side by side were evaluated by a radiologist and a nuclear medicine specialist in consensus. $\mathrm{PET} / \mathrm{CT}$ and $\mathrm{PET}+\mathrm{CT}$ readings were performed at least 2 months apart.

The participating readers were informed about the patient-specific clinical background, but blinded to the results of the other imaging procedures and the results of the clinical investigation (e.g., endoscopy).

Lesion detection on combined PET/CT and PET $+\mathrm{CT}$ was based on the detection of soft tissue masses with contrast enhancement in conjunction with a focally increased glucose metabolism above the surrounding tissue level. Furthermore, a maximum standardised uptake value (SUVmax) of at least more than 2.5 supported the diagnosis of a malignant lesion. The PET threshold was adjusted to $40 \%$ of the maximum SUV, which defines the size of the metabolically active lesion [21, 22].

The T stage was evaluated based on the tumour size and surrounding tissue infiltration with corresponding elevated glucose metabolism on PET/CT and PET $+\mathrm{CT}$. Lymph nodes were assessed for metastatic spread based on an increased glucose metabolism and independent of their size on PET/CT and PET+CT.

Distant metastases were assessed based on the detection of soft tissue masses with contrast enhancement in different body compartments and in conjunction with focally increased glucose metabolism above the surrounding tissue level. The diagnosis of a distant metastasis was furthermore supported by an SUVmax of at least 2.5 (extrahepatic) and 3.5 (intrahepatic) [23]. In cases of malignant findings on CT-only without focally increased glucose metabolism, the lesions were evaluated based on CT criteria (see below).
On CT-only images, detection of soft tissue masses with contrast enhancement characterised malignancy. The T stage was assessed based on lesion size, surrounding tissue infiltration, infiltration of other organs and the localisation of the mass. The lymph node assessment was based on lesion size: a threshold of $1.5 \mathrm{~cm}$ and $1.0 \mathrm{~cm}$ was used for jugulodigastric lymph nodes and all other lymph nodes of the neck, respectively [24]. The overall TNM-stage for all tumour types was assessed based on the current AJCC criteria for all imaging procedures except PET imaging [25].

\section{Lesion detection}

Lesions detection was compared for all imaging modalities (CT, PET, PET $+\mathrm{CT}$ and PET/CT) to show comparability with the available literature and to demonstrate differences of importance of lesion detection and TNM staging. CT, $\mathrm{PET}+\mathrm{CT}$ and PET/CT images were evaluated for lesion detection based on the above mentioned criteria.

PET-only images were evaluated quantitatively and qualitatively for areas of focally increased glucose metabolism above the surrounding tissue level, supported by a SUVmax $\geq 2.5$ (excluding brain). However, PET-only images were used to assess lesion detection rates, but not TNM staging. This approach was chosen, because PETonly imaging is not accepted as a primary imaging staging procedure for head and neck cancer $[2,6,7]$. Volume and size measurement of head and neck cancer based on PETonly is a subject of great dispute, because T- and N-stage evaluation can be impaired by the limited anatomical resolution of the PET, thus rendering a surrounding tissue infiltration, for example, challenging [26]. Hence, the PET evaluation was restricted to lesion detection alone (sensitivity, specificity, accuracy, NPV, PPV).

\section{Change in management}

The impact on patient management concerning the differences of the imaging results was assessed by the referring physicians and the radiologists and nuclear medicine specialists in consensus and was based on the international clinical guidelines [6].

\section{Standard of reference}

In all patients histopathological evaluation and the resected surgical specimen (tumours and/or lymph nodes) served as the standard of reference for both $\mathrm{T}$ - and N-stage. Patients who had a lymphoma only had biopsy. A mean follow-up time of 407 days (range: 7-1,581 days) served as the standard of reference for M0-staged patients. Short followup times $(<100$ days) in two patients were due to benign rather than malignant findings. Patients with M1 stage had 
histopathological confirmation of the suspected distant metastases.

\section{Statistical analysis}

The primary endpoint of this study was the correct classification of the overall TNM stage using CT and $\mathrm{PET}+\mathrm{CT}$ in comparison to PET/CT. Differences in TNM staging between the different imaging procedures were tested for significance using the exact McNemar's test. Bonferroni correction was applied to account for multiple comparisons. The nominal significance level to evaluate the two hypotheses of the primary analysis has to be 0.025 to maintain a global significance level of 0.05 . We calculated 95\% confidence intervals (CI) according to Tango for the difference in correlated proportions of the correct TNM tumour stage [27].

Differences in the assessment of the T, N, and M-stages between the imaging procedures (secondary endpoints) were tested for significance using the exact McNemar's test with a significance level of 0.05 .

Further endpoints included the determination of sensitivities, specificities, negative predictive values (npv), positive predictive values (ppv), and accuracies (with exact $95 \%$ confidence intervals) for tumour detection, for lymph node detection, and distant metastases detection in all modalities using histology as the standard of reference. Statistical analyses were performed with SAS statistical software (Version 9.1, SAS Institute Inc., Cary, NC).

\section{Results}

\section{Patients}

Fifty-five consecutive patients in a university hospital setting underwent combined PET/CT imaging and were included in this retrospective study.

The definitive diagnoses of tumour entities and/or benign lesions were based on the standard of reference and are listed in Table 1. The TNM-staging evaluation was based on 31 operated patients, respectively. Six patients with lymphoma were excluded, as well as four patients with inflammatory lesions (including one patient with lymph node tuberculosis), and two patients with benign tumours. In 12 patients no primary tumour was found (CUP: carcinoma of unknown primary). In these patients, neck dissection based on the PET/CT findings served as the standard of reference.

Overall accuracy of the different imaging procedures for detection of malignant tumours was assessed based on 25 patients with primary tumour resection and additional neck dissection; 6 patients had primary tumour resection only. All patients tolerated the PET/CT procedure well.
Table 1 Definitive diagnosis after surgery and/or neck dissection and biopsy

\begin{tabular}{ll}
\hline Definitive diagnoses (tumour entities and benign lesions) & Patients \\
\hline Carcinoma of the oral cavitiy & 9 \\
Carcinoma of the nasopharynx & 1 \\
Carcinoma of the oropharynx & 5 \\
Carcinoma of the hypopharynx & 3 \\
Esophagus carcinoma & 1 \\
Carcinoma of the larynx & \\
-Supraglottic & 7 \\
-Glottic & 4 \\
Carcinoma of the outer ear & 1 \\
Carcinoma of unknown primary & 12 \\
Hodgkin's disease & 2 \\
Non-Hodgkin's lymphoma & 4 \\
Inflammatory lesions & 3 \\
Lymph node tuberculosis & 1 \\
Nasopharyngeal cyst & 1 \\
Choanal/pharyngeal polyp & 1 \\
Total & 55 \\
\hline
\end{tabular}

Six patients only received biopsy and did not undergo additional surgery based on histopathological findings (lymphoma).

\section{TNM staging}

Concerning the definition of the overall TNM-stage, combined FDG-PET/CT imaging was significantly more accurate ( $\mathrm{p}<0.05$; nominal significance level) than CT-only (difference $55 \% ; 95 \%$ CI: $38 \%-71 \%$ ) and $\mathrm{PET}+\mathrm{CT}$ (difference $32 \%$; 95\% CI: $18 \%-50 \%)(\mathrm{p}<0.05)$. The results were based in parts PET/CT yielding a more accurate T-stage (difference 65\%; 95\% CI: 46\%-79\%, p<0.05) (Fig. 1) and a marginally more accurate N-stage (difference $29 \%$; $95 \%$ CI: $4 \%-41 \%)(p=0.04)$ when compared to CT-only (Fig. 2). However, compared to PET $+\mathrm{CT}$ the improvements in Tstaging from PET/CT were smaller (difference $32 \%$; $95 \% \mathrm{CI}$ : $18 \%-50 \%)(\mathrm{p}<0.05)$; no significant difference was found for N-stage (difference 13\%; 95\% CI: 0\%-29\%) ( $>>0.05$ ) (Fig. 3). Based on a patient-by-patient analysis, T-stage was correctly determined in $27 / 31$ patients $(87 \%$; CI: $70 \%-96 \%)$, the N-stage in $24 / 31$ patients (77\%; CI: $59 \%-90 \%)$ and the $\mathrm{M}$ stage in 30/31 patients (97\%; CI: 83\%-100\%) with PET/CT. The T-stage was correctly determined in $17 / 31$ patients $(55 \%$; CI: $36 \%-73 \%)$, the N-stage in 20/31 patients (65\%; CI: $45 \%-$ $81 \%$ ) and the M-stage in 30/31 patients (97\%; CI: $83 \%-$ $100 \%$ ) based on evaluation with PET $+\mathrm{CT}$. The T-stage was correctly determined in $7 / 31$ patients $(23 \%$; CI: $10 \%-41 \%)$, the N-stage in $17 / 31$ patients (55\%; CI: $36 \%-73 \%$ ) and the M-stage in 30/31 patients (97\%; CI: $83 \%-100 \%$ ) when evaluated with CT-only. Consequently, combined PET/CT imaging leads to fewer cases with under- or overestimated TNM stages compared to the other imaging modalities. $A$ summary of all TNM staging results is shown in Table 2. 


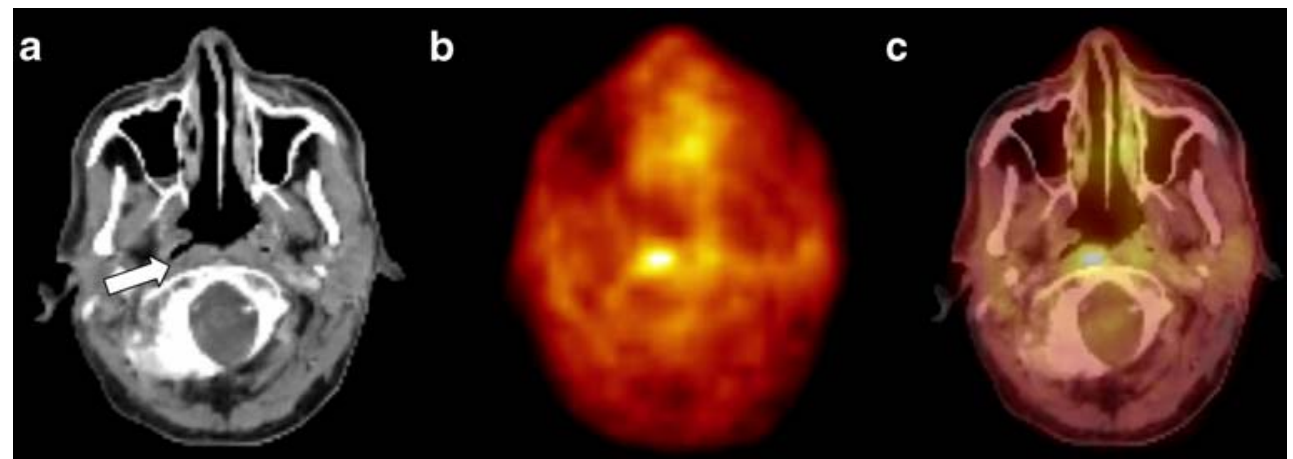

Fig. 1 A 56-year-old male patient with suspected epipharyngeal cancer. (a) Axial CT image shows symmetric pharyngeal structures in a patient with suspected epipharyngeal cancer. Furthermore, no suspicious contrast enhancement or thickening of the dorsal epipharyngeal wall (white arrow) or other pharyngeal wall structures were detected. Thus, on CT images, the tumour was overlooked by the reader team. (b) Corresponding axial PET image of the same area shows a focally increased glucose metabolism at the right epipharyngeal wall. On PET images alone, exact localisation of the increased

\section{Patients with CUP and benign lesions}

No primary tumour was found in 12 patients with suspected head and neck cancer. Consequently, these patients were classified as CUP patients. When evaluating the N-stage and M-stage in these 12 CUP patients, 1 patient was overstaged, 1 patient was understaged and 10 patients were correctly staged with PET/CT, whereas 2 patients were overstaged, 1 patient was understaged and 9 patients were correctly staged with $\mathrm{PET}+\mathrm{CT}$ compared to the standard of reference. When staged with CT-only, one patient was overstaged, four patients were understaged and seven patients were staged correctly, respectively.

Furthermore, several benign or non-malignant lesions were detected (one osteomyelitis at the base of the skull, one tonsilitis, one clotted internal jugular vein with surrounding inflammation, one lymph node tuberculosis, glucose metabolism is challenging. However, on PET images, the tumour was detected (true-positive result). (c) Corresponding axial PET/CT image shows an area of increased glucose metabolism in the non-thickened right epipharyngeal wall. Furthermore, there is an asymmetric distribution of tracer activity compared to the left tonsil. Thus, on PET/CT images, the tumour was correctly detected, localised and characterised. The T-stage was confirmed by subsequent surgical resection and histopathological workup

one cyst and one choanal/pharyngeal polyp). All modalities (CT-only, PET $+\mathrm{CT}$ and PET/CT) were evaluated false positive in one patient with lymph node tuberculosis. In one case with osteomyelitis at the base of the skull the dorsal nasopharyngeal wall was thickened and showed a high consecutive FDG uptake. These findings were falsely interpreted as tumour by all readers in all modalities.

\section{Lesion detection}

Combined FDG-PET/CT showed a higher detection rate (97\%; CI: 83\%-100\%) than PET+CT (90\%; CI: 74\%-98\%/ p $>0.05)$, CT-only ( $58 \%$; CI: $39 \%-75 \%, p<0.05)$ and PETonly (87\%; CI: $70 \%-96 \%, \mathrm{p}>0.05)$ for the detection of the primary tumour. Hence, a statistical significant difference was detected only when compared to CT-only. However, assessing lymph node detection, PET/CT and PET $+\mathrm{CT}$

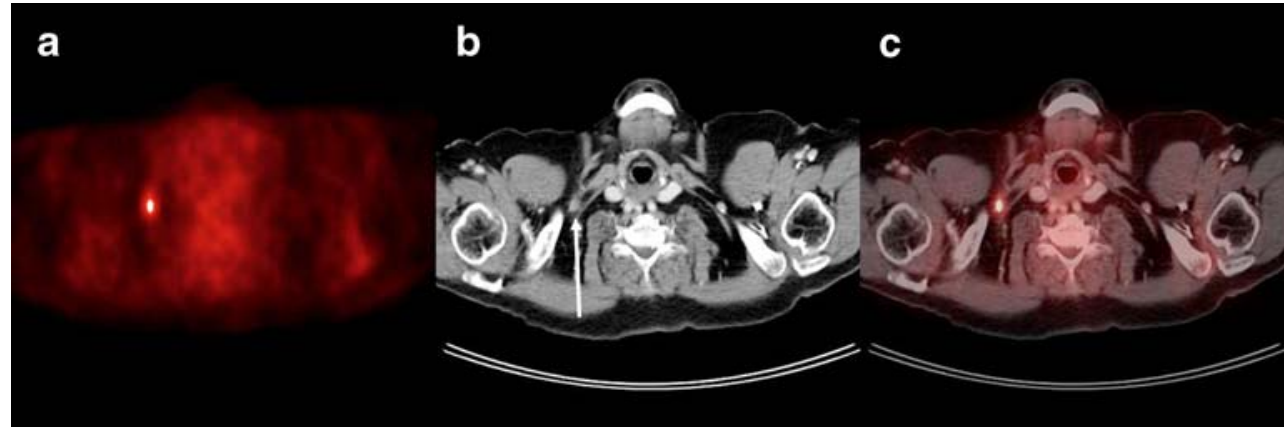

Fig. 2 An 83-year-old female patient with suspected oropharyngeal cancer. (a) Axial PET image shows an area of increased glucose metabolism right cervically/retroclavicularly. However, no other area with a pathologically increased glucose metabolism could be detected in any pharyngeal section (not shown). (b) Axial CT of the same patient shows a 5-mm lesion (white arrow). On CT alone this small lymph node was considered not malignant. On side-by-side viewing, the lymph node was detected and evaluated as suspicious for metastatic spread. (c) Fused PET/CT detected elevated glucose metabolism within this small lymph node. Consecutive resection confirmed metastatic spread in this lymph node. However, also on $\mathrm{PET} / \mathrm{CT}$ no primary tumour could be detected in any pharyngeal section. Thus, in this patient the diagnosis remained CUP 


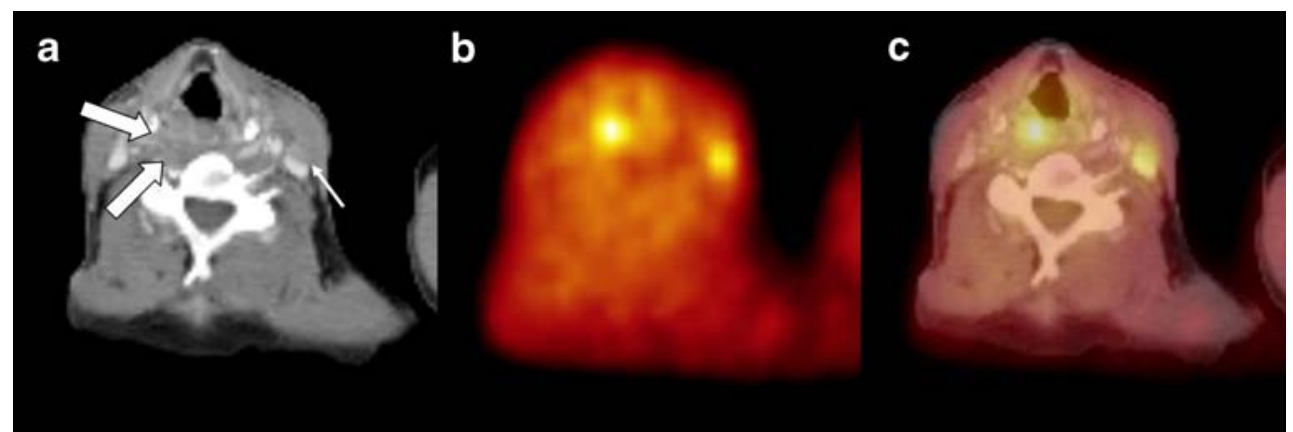

Fig. 3 A 67-year-old female patient with suspected supraglottic, laryngeal cancer. (a) The axial CT image shows a nodular, thickened pharyngeal/laryngeal wall (bold white arrows). A small lymph node left cervically was overlooked based on its size and proximity to the M. sternocleidomastoideus (thin white arrow). (b) The axial PET image shows an elevated glucose metabolism of the pharyngeal/ laryngeal wall. Furthermore, a small area with elevated glucose

yield similar diagnostic accuracies and no statistically significant difference was found to PET-only and CT-only. Table 3 summarizes the overall detection rates for the primary tumour and lymph node metastases.

\section{Therapy alteration}

The impact on patient therapy was assessed based on international clinical guidelines [6]. In seven patients, there was additional information provided by combined PET/CT

Table 2 TNM staging results

\begin{tabular}{llll}
\hline & $\begin{array}{l}\text { Correctly staged } \\
\text { patients/95\%-CI }\end{array}$ & $\begin{array}{l}\text { Overstaged } \\
\text { patients }\end{array}$ & $\begin{array}{l}\text { Understaged } \\
\text { patients }\end{array}$ \\
\hline TNM stage & & & \\
PET/CT & $34 / 49 / 55 \%-82 \%$ & $6 / 49$ & $9 / 49$ \\
PET+CT & $23 / 49 / 33 \%-62 \%$ & $7 / 49$ & $19 / 49$ \\
CT & $15 / 49 / 18 \%-45 \%$ & $7 / 49$ & $27 / 49$ \\
T stage & & & \\
PET/CT & $44 / 49 / 78 \%-97 \%$ & $2 / 49$ & $3 / 49$ \\
PET+CT & $32 / 49 / 50 \%-78 \%$ & $4 / 49$ & $13 / 49$ \\
CT & $24 / 49 / 34 \%-64 \%$ & $5 / 49$ & $20 / 49$ \\
N stage & & & \\
PET/CT & $38 / 49 / 63 \%-88 \%$ & $5 / 49$ & $6 / 49$ \\
PET+CT & $34 / 49 / 55 \%-82 \%$ & $8 / 49$ & $7 / 49$ \\
CT & $30 / 49 / 46 \%-75 \%$ & $7 / 49$ & $12 / 49$ \\
M stage & & & \\
PET/CT & $48 / 49 / 89 \%-100 \%$ & $1 / 49$ & $0 / 49$ \\
PET+CT & $47 / 49 / 86 \%-100 \%$ & $2 / 49$ & $0 / 49$ \\
CT & $48 / 49 / 89 \%-100 \%$ & $0 / 49$ & $1 / 49$ \\
\hline
\end{tabular}

Staging results with the different imaging modalities for the overall TNM-stage, for the T-stage, the N-stage, and the M-stage including overstaging and understaging

Forty-nine represents the total number of patients including benign lesions and CUP patients. Six patients of the total study population had to be excluded from TNM-staging as they were found to have lymphoma $(95 \% \mathrm{CI})$ metabolism was detected left cervically. Thus, on side-by-side imaging the tumour as well as the lymph node metastasis were detected. (c) Combined PET/CT shows the tumour with elevated glucose metabolism right cervically as well as the small lymph node with elevated glucose metabolism left cervically. PET/CT was correct in tumour detection and lymph node detection. Hence, PET/CT was superior in lymph node detection over CT alone, but not over PET $+\mathrm{CT}$

not afforded by PET $+\mathrm{CT}$ side by side, which altered the therapeutical management (7/31 patients, 23\%). Compared to $\mathrm{PET}+\mathrm{CT}$, PET/CT-based therapy changes derived from upstaging in six patients with consecutive alteration of the surgical extent. This was based on six patients with more accurate T-stage evaluation, and no therapy alteration was achieved by more accurate lymph node evaluation. PET/CT allowed for correct downstaging in one patient, thus, preventing the patient from unnecessary surgery. Staging based on combined PET/CT imaging altered the therapy in $13 / 31$ patients $(42 \%)$ compared to CT-only. In eight of these patients the T-stage was characterised more accurately with $\mathrm{PET} / \mathrm{CT}$, thus resulting in an increase of the surgical extent. In one patient, FDG-PET/CT was able to upstage both Tstage and $\mathrm{N}$-stage, leading to more extensive surgery. Correct downstaging was possible in four patients when comparing PET/CT to CT-only readings, thus, reducing the extent of the surgery for the primary tumour. Since there was only a marginally significantly more accurate lymph node evaluation ( $\mathrm{p}=0.04)$ with PET/CT vs. CT-only, no change in therapy was found concerning the $\mathrm{N}$-stage evaluation.

\section{Discussion}

Whole-body PET/CT with a dedicated, contrast-enhanced head and neck protocol proved to be partly significantly superior in overall TNM staging of suspected primary head and neck cancers compared to PET $+\mathrm{CT}$ and compared to contrast-enhanced CT-only. These results were mainly based on a significantly more accurate T-staging $(\mathrm{p}<0.05)$ compared to $\mathrm{PET}+\mathrm{CT}$ and $\mathrm{CT}$-only based on better visibility in patients with metallic oral implants. Only a barely more accurate $\mathrm{N}$-staging $(\mathrm{p}=0.04)$ compared to CTonly was found. However, PET/CT changed the therapy in a substantial number of patients compared to the compared 
Table 3 Lesion detection

\begin{tabular}{|c|c|c|c|c|c|c|c|c|c|c|}
\hline & $\begin{array}{l}\text { Sensitivity } / 95 \%- \\
C I\end{array}$ & $\mathrm{n}$ & $\begin{array}{l}\text { Specificity } / 95 \%- \\
C I\end{array}$ & $\mathrm{n}$ & $\begin{array}{l}\text { Accuracy } / 95 \%- \\
C I\end{array}$ & $\mathrm{n}$ & $\mathrm{PPV} / 95 \% C I$ & $\mathrm{n}$ & $\mathrm{NPV} / 95 \% C I$ & $\mathrm{n}$ \\
\hline \multicolumn{11}{|c|}{ A. Tumour detection } \\
\hline $\mathrm{PET} / \mathrm{CT}$ & $97 \% / 83 \%-100 \%$ & 31 & $94 \% / 73 \%-100 \%$ & 18 & $96 \% / 86 \%-100 \%$ & 49 & $97 \% / 83 \%-100 \%$ & 31 & $94 \% / 73 \%-100 \%$ & \\
\hline $\mathrm{PET}+\mathrm{CT}$ & $90 \% / 74 \%-98 \%$ & 31 & $83 \% / 59 \%-96 \%$ & 18 & $88 \% / 75 \%-95 \%$ & 49 & $90 \% / 74 \%-98 \%$ & 31 & $83 \% / 59 \%-96 \%$ & \\
\hline $\mathrm{CT}$ & $58 \% / 39 \%-75 \%$ & 31 & $94 \% / 73 \%-100 \%$ & 18 & $71 \% / 57 \%-83 \%$ & 49 & $95 \% / 74 \%-100 \%$ & 19 & $57 \% / 37 \%-75 \%$ & 30 \\
\hline PET & $87 \% / 70 \%-96 \%$ & 31 & $83 \% / 59 \%-96 \%$ & 18 & $86 \% / 73 \%-94 \%$ & 49 & $90 \% / 73 \%-98 \%$ & 30 & $79 \% / 54 \%-94 \%$ & \\
\hline \multicolumn{11}{|c|}{ B. Lymph node detection } \\
\hline $\mathrm{PET} / \mathrm{CT}$ & $87 \% / 70 \%-96 \%$ & 31 & $79 \% / 58 \%-93 \%$ & 24 & $84 \% / 71 \%-92 \%$ & 55 & $84 \% / 67 \%-95 \%$ & 32 & $83 \% / 61 \%-95 \%$ & 2. \\
\hline $\mathrm{PET}+\mathrm{CT}$ & $87 \% / 70 \%-96 \%$ & 31 & $67 \% / 45 \%-84 \%$ & 24 & $78 \% / 65 \%-88 \%$ & 55 & $77 \% / 60 \%-90 \%$ & 35 & $80 \% / 56 \%-94 \%$ & 20 \\
\hline $\mathrm{CT}$ & $77 \% / 59 \%-90 \%$ & 31 & $75 \% / 53 \%-90 \%$ & 24 & $76 \% / 63 \%-87 \%$ & 55 & $80 \% / 61 \%-92 \%$ & 30 & $27 \% / 51 \%-88 \%$ & 25 \\
\hline PET & $81 \% / 63 \%-93 \%$ & 31 & $71 \% / 49 \%-87 \%$ & 24 & $76 \% / 63 \%-87 \%$ & 55 & $78 \% / 60 \%-91 \%$ & 32 & $74 \% / 52 \%-90 \%$ & 23 \\
\hline \multicolumn{11}{|c|}{ C. Distant metastases detection } \\
\hline $\mathrm{PET} / \mathrm{CT}$ & $100 \% / 29 \%-100 \%$ & 3 & $98 \% / 88 \%-100 \%$ & 46 & $98 \% / 89 \%-100 \%$ & 49 & $75 \% / 19 \%-99 \%$ & 4 & $\begin{array}{c}100 \% / 92 \%- \\
100 \%\end{array}$ & 4 \\
\hline $\mathrm{PET}+\mathrm{CT}$ & $100 \% / 29 \%-100 \%$ & 3 & $96 \% / 85 \%-99 \%$ & 46 & $96 \% / 86 \%-100 \%$ & 49 & $60 \% / 15 \%-95 \%$ & 5 & $\begin{array}{c}100 \% / 92 \%- \\
100 \%\end{array}$ & \\
\hline $\mathrm{CT}$ & $67 / 9 \%-100 \%$ & 3 & $100 \% / 92 \%-100 \%$ & 46 & $98 \% / 89 \%-100 \%$ & 49 & $\begin{array}{c}100 \% / 16 \%- \\
100 \%\end{array}$ & 2 & $98 \% / 89 \%-100 \%$ & 47 \\
\hline PET & $33 \% / 1 \%-91 \%$ & 3 & $93 \% / 82 \%-99 \%$ & 46 & $90 \% / 78 \%-97 \%$ & 49 & $25 \% / 1 \%-81 \%$ & 4 & $96 \% / 85 \%-99 \%$ & \\
\hline
\end{tabular}

Rates for primary tumour detection (Table 4A), lymph node detection (Table 4B), and detection of distant metastases (Table 4C). Patients with cervical lymphoma have been included for evaluation of lymph node detection $(n=55)$

PPV: positive predictive value, NPV: negative predictive value, CI: confidence interval; $n=$ number of patients evaluation is based on

imaging modalities. Thus, our study suggests that staging, rather than lesion detection only, is important for therapy influence. Overall, PET/CT therefore may be considered as the first line diagnostic option in staging patients with suspected primary head and neck cancer.

\section{Lesion detection}

Lesions detection was compared for all lesions and all imaging modalities (CT, PET, PET $+\mathrm{CT}$ and PET/CT) to show comparability with the available literature and to demonstrate differences of importance of lesions detection and TNM-staging.

\section{PET/CT vs. PET}

Although cross-sectional imaging has been recommended as the first-line tool for tumour detection in the head and neck area, PET has already shown outstanding results concerning staging of head and neck cancers with focus on tumour detection and lymph node assessment $[9,11,28$, 29]. Our results concerning lymph node detection and detection of the primary tumour are well in accordance with the current literature. When comparing overall lesion detection (primary tumour and metastatic lymph nodes) of $\mathrm{PET}$ and PET/CT, comparable studies indicated sensitivities of $67 \%-93 \%$ and up to $100 \%$ for the specificity with PET, respectively $[8,12,30]$. For PET/CT, sensitivities of up to
$100 \%$ and specificities ranging from $77 \%-92 \%$ have been reported $[13,14,31]$. In the head and neck area, the complex anatomy and the varying physiological uptake of FDG renders PET-only interpretation difficult and challenging $[15,32,33]$. The availability of complementary CTdata in PET/CT may help to overcome those interpretation difficulties.

\section{PET/CT vs. CT}

For detection of malignancy with $\mathrm{CT}$, we found a relatively low sensitivity, but high specificity for primary tumour detection and reasonable results for lymph node detection. Lesion detection rates have been discussed controversially for CT with reported sensitivities and specificities ranging from $25 \%-74 \%$ and $25 \%-75 \%$ for the primary lesion and up to $97 \%$ sensitivities and specificities for lymph node detection, respectively $[13,30,34,35]$. One reason for the differences may be that most of the other studies included an inhomogeneous patient population evaluating primary and recurrent cancers, while we concentrated on primary cancers in patients who had not received previous treatment. Additionally, CT staging can be heavily impacted by metallic implants, as this was present in a significant number of patients in our population. In those cases, additional functional information can be of substantial help. Overall, CT staging in this particular clinical setting was not as reliable, especially in T-staging, as combined imaging. 


\section{PET/CT vs. PET+CT}

We noticed only minor differences when comparing PET/ $\mathrm{CT}$ and PET $+\mathrm{CT}$ for lymph node detection and M-staging. Therefore, PET $+\mathrm{CT}$ might be generally considered a sufficient alternative. Several issues have to be kept in mind when comparing two dedicated procedures with an integrated PET/CT: despite the logistical and time-consuming disadvantages for the patient, the referring physician has to combine two reports on his/her own. Being a clinical physician, this might be time consuming and sometimes challenging, too. Furthermore, those similar results might be overestimated for several reasons (see limitations).

\section{TNM-staging and therapy alterations}

Only a few studies have been published concerning combined imaging with PET/CT in head and neck cancer and mainly lesion detection and positive effects of PET/CT on localisation over CT alone and PET alone were evaluated rather than TNM-staging. In one study, Schoeder and co-workers defined the accuracy of PET/CT in N0staged cervical lymph nodes defined by clinical examination, CT and MRI. Compared to our results, a lower sensitivity, but a higher specificity and a comparable accuracy were found [16]. In this study, the surgical plan was not influenced by the PET/CT- findings, because risk stratification was already based on the conventional workup. The difference in our study is that the PET/CT in our centre was already accepted as the standard of care by the operating physician because it contained a contrastenhanced CT. Therefore, we found a higher diagnostic impact.

In another study, Schoeder et al. evaluated the reader confidence defined by a qualitative score. They found a higher diagnostic confidence with consecutive affected patient care based on the PET/CT findings, but therapeutic treatment was altered in 4 of 68 patients when compared to PET-only [29]. Thus, the effect on patient care was mainly due to additional imaging studies, which could be avoided by the higher diagnostic confidence. In a recent study by Connell et al. the TNM classification was altered by PET/ $\mathrm{CT}$ when compared to the conventional clinical workup in $34 \%$, representing a similar range compared to our findings [36]. Moreover, the radiotherapy was influenced in $29 \%$ of the patients based on PET/CT findings. Again, our results, even retrospectively evaluated, are showing the same range of influence on therapy management. However, in discrepancy to our study, histopathological verification of the malignant findings were not commonly optained.

Branstetter et al. evaluated the improvement of reader confidence concerning the lesion detection and characterisation, too. They found an improvement in $62 \%$ of lesions compared to CT alone and in $41 \%$ of lesions compared to PET alone [13]. Our results point out a similar trend. Overall, the combination of increased reader confidence (i.e., more lesions were detected and characterised as malignant than in CT-only) and the additional information afforded by the PET-component mainly contributed to the therapeutical influence of the PET/CT findings in our study.

In our study, PET/CT correctly changed consecutive therapy in a substantial number of patients compared to $\mathrm{CT}$ alone and compared to $\mathrm{PET}+\mathrm{CT}$. Alterations of patient management mainly derived from a more accurate overall TNM-staging and mainly upstaging. Therapy alterations, as evaluated in this study, were based on standard clinical guidelines, but were conducted retrospectively. These therapy alterations must therefore be considered "potential" therapy alterations, because, in prospective evaluations, individual decisions of the patient and/or the physician can lead to different therapies. However, these retrospective evaluations still represent clinical routine, at least to a certain extent. Although we could demonstrate an advantage in TNM-staging and showed therapy changes based on PET/CT compared to the other modalities, it still needs to be proven if these therapeutic influences have an effect on survival or quality of live.

\section{Limitations}

\section{Limiting factors: PET/CT imaging}

There are some limitations to the PET/CT procedure: Metallic implant artefacts not only can cause difficulties in CT reading, but also in PET/CT due to artefacts caused by the CT-based PET attenuation correction. However, in those cases, evaluation of the non-corrected PET images are of additional help. Therefore, PET/CT can overcome classical CT-based evaluation difficulties, especially in patients with disturbing metallic oral implants.

CT-only imaging in this study arose from the combined PET/CT acquisition. Thus, resolution was limited based on the technical capabilities of the used dual-slice scanner. In reality, state-of-the-art multi-slice CT scanners meanwhile can provide much higher resolution with only slightly increased noise, providing (together with a dedicated contrast media protocol) a significantly higher reader confidence. Furthermore, a dedicated head and neck CT is generally conducted with an angled (parallel to the base of the skull) acquisition projection and a different contrast media protocol. Due to geometrical limitations of the PET/CT, tilting of the gantry is not feasible for PET/CT. These non-angled CT images may have an impact on the detection of small lesions, especially at the base of the skull.

Based on a varying glucose uptake in the head and neck area, detection and staging of malignant lesions can be challenging $[33,37]$. Especially the tonsils, as well as the vocal cords, may show a high SUV, leading to false-positive 
findings [32, 33]. Inflammation is another major pitfall in evaluation of ENT cancer. In our patient population, two patients were evaluated false positive based on inflammatory lesions, too. Different approaches to differentiate between inflammation and tumour with dual-time PET or alternative tracers have shown promising results, but have not been implemented in clinical routine [8, 38].

\section{Limiting factors: study design}

The study design itself has some limitations. Only a small number of patients are included in our study at first sight. However, it has to be noted that only patient with primary suspicion of head and neck cancer and with a complete histopathological workup were included in our study. Certainly, significantly more patients with head and neck malignancies are examined in our institution, but a significant number of patients are treated with radiotherapy and/or chemotherapy rather than primary surgery. In these patients, only a mixed standard of reference (histopathology, clinical course, laboratory reports, imaging follow-up) was available. As one aim of the study was to compare the imaging modalities concerning TNM-staging with the most adequate standard of reference (histopathology), primary tumour operation and/or neck dissection conducted before any additional therapy was needed. Thus, a large number of patients had to be excluded. However, false-negative lymph nodes could partly only be evaluated based on clinical follow-up, because neck dissection was not always performed in patients where no cervical lymph node metastases were suspected.

In clinical routine, pre-therapeutic staging is not only based on imaging findings, but also on endoscopical procedures. The combination of endoscopical procedures with PET/CT imaging will probably serve as the most sophisticated approach for staging of head and neck tumours. Small, but important findings on endoscopy (e.g., fixation of the vocal cord) have additional impact on T-staging. PET/CT then may provide complementary $\mathrm{N}$-staging and M-staging for further therapy planning.

Patients with CUP (carcinoma of unknown primary) were also evaluated in our study. It has been reported in the literature that in some patients with CUP, no primary tumour will be detected during the course of the disease with any diagnostic method [16, 39]. In our study, the primary tumour was not found during the clinical follow-up in those patients as well; thus, they had been excluded from the overall TNM evaluation as well.

Finally, minor differences were detected when comparing tumour staging with $\mathrm{PET} / \mathrm{CT}$ and $\mathrm{PET}+\mathrm{CT}$. In clinical routine, $\mathrm{CT}$ and PET for side-by-side evaluation used to be acquired on different scanners, leading to different patient positioning on the examination tables, different organ positions based on respiration and organ shift and based on different fields of view. We tried to mimic those situations by manual misalignment of CT and PET on two different screens. This might be the reason why PET/CT performed significantly better in T-staging evaluation than $\mathrm{PET}+\mathrm{CT}$. Therefore, in clinical routine, side-by-side image evaluation, when acquired separately on different scanners, remains challenging and will probably be less accurate than combined PET/CT. Additionally, the vast majority of scanners that are actually sold are PET/CT-scanners, rather than PET-only scanners.

\section{Conclusion}

Contrast-enhanced FDG-PET/CT was partly superior in TNM-staging of head and neck cancer compared to contrast-enhanced CT-only and PET $+\mathrm{CT}$. This was based on a more reliable T-staging, partly due to better primary tumour delineation on co-registered $\mathrm{PET} / \mathrm{CT}$ in patients with metallic artefacts. PET/CT could lead to a change in patient management in a substantial number of patients when compared to CT-only and PET+CT. PET/CT, PET+CT and PET-only performed equally well for lesion detection. In summary, contrast-enhanced, combined FDG-PET/CT imaging might be considered appropriate to replace single CTand PET imaging for staging head and neck tumours.

\section{References}

1. Jemal A, Murray T, Ward E, Samuels A, Tiwari RC, Ghafoor A, et al. Cancer statistics, 2005. CA Cancer J Clin 2005;55(1):10-30.

2. NCCN. http://www.ncen.org. In: National Comprehensive Cancer Network.

3. Caponigro F, Milano A, Basile M, Ionna F, Iaffaioli RV. Recent advances in head and neck cancer therapy: the role of new cytotoxic and molecular-targeted agents. Curr Opin Oncol 2006;18(3):247-52.

4. Cohen EE, Rosen F, Stadler WM, Recant W, Stenson K, Huo D, et al. Phase II trial of ZD1839 in recurrent or metastatic squamous cell carcinoma of the head and neck. J Clin Oncol 2003;21(10): 1980-7.

5. Colevas AD. Chemotherapy options for patients with metastatic or recurrent squamous cell carcinoma of the head and neck. J Clin Oncol 2006;24(17):2644-52.

6. AWMF. http://www.awmf-online.de.

7. ESMO. http://www.esmo.org. In.

8. Pauleit D, Zimmermann A, Stoffels G, Bauer D, Risse J, Fluss MO, et al. 18F-FET PET Compared with 18F-FDG PET and CT in Patients with Head and Neck Cancer. J Nucl Med 2006;47(2): 256-61.

9. Wong WL, Saunders M. The impact of FDG PET on the management of occult primary head and neck tumours. Clin Oncol (R Coll Radiol) 2003;15(8):461-6.

10. Yousem DM, Montone KT. Head and neck lesions. Radiologicpathologic correlations. Radiol Clin North Am 1998;36(5):9831014, vii.

11. Kau RJ, Alexiou C, Laubenbacher C, Werner M, Schwaiger M, Arnold W. Lymph node detection of head and neck squamous cell 
carcinomas by positron emission tomography with fluorodeoxyglucose F 18 in a routine clinical setting. Arch Otolaryngol Head Neck Surg 1999;125(12):1322-8.

12. Wong WLCE, McGurk M, Hussain K, Davis J, Beaney R, Baddeley $\mathrm{H}$, et al. A prospective study of PET-FDG imaging for the assessment of head and neck squamous cell carcinoma. Clin Otolaryngol Allied Sci 1997;22(3):209-14.:209-14.

13. Branstetter BFt, Blodgett TM, Zimmer LA, Snyderman CH, Johnson JT, Raman S, et al. Head and neck malignancy: is PET/CT more accurate than PET or CT alone? Radiology 2005;235(2):580-6.

14. Goshen E, Davidson T, Yahalom R, Talmi YP, Zwas ST. PET/CT in the evaluation of patients with squamous cell cancer of the head and neck. Int J Oral Maxillofac Surg 2006;35(4):332-6.

15. Ha PK, Hdeib A, Goldenberg D, Jacene H, Patel P, Koch W, et al. The role of positron emission tomography and computed tomography fusion in the management of early-stage and advanced-stage primary head and neck squamous cell carcinoma. Arch Otolaryngol Head Neck Surg 2006;132(1):12-6.

16. Schoder H, Carlson DL, Kraus DH, Stambuk HE, Gonen M, Erdi YE, et al. 18F-FDG PET/CT for detecting nodal metastases in patients with oral cancer staged N0 by clinical examination and CT/MRI. J Nucl Med 2006;47(5):755-62.

17. Antoch G, Jentzen W, Freudenberg LS, Stattaus J, Mueller SP, Debatin JF, et al. Effect of oral contrast agents on computed tomography-based positron emission tomography attenuation correction in dual-modality positron emission tomography/computed tomography imaging. Invest Radiol 2003;38(12):784-9.

18. Antoch G, Kuehl H, Kanja J, Lauenstein TC, Schneemann H, Hauth E, et al. Dual-modality PET/CT scanning with negative oral contrast agent to avoid artifacts: introduction and evaluation. Radiology 2004;230(3):879-85.

19. Beyer T, Antoch G, Blodgett T, Freudenberg LF, Akhurst T, Mueller S. Dual-modality PET/CT imaging: the effect of respiratory motion on combined image quality in clinical oncology. Eur J Nucl Med Mol Imaging 2003;30(4):588-96.

20. Beyer T, Tellmann L, Nickel I, Pietrzyk U. On the use of positioning aids to reduce misregistration in the head and neck in whole-body PET/CT studies. J Nucl Med 2005;46(4):596-602.

21. Biehl KJ, Kong FM, Dehdashti F, Jin JY, Mutic S, El Naqa I, et al. 18F-FDG PET definition of gross tumor volume for radiotherapy of non-small cell lung cancer: is a single standardized uptake value threshold approach appropriate? J Nucl Med 2006;47(11):1808-12.

22. Drever L, Roa W, McEwan A, Robinson D. Iterative threshold segmentation for PET target volume delineation. Med Phys 2007;34(4):1253-65.

23. Delbeke D, Martin WH, Sandler MP, Chapman WC, Wright JK Jr, Pinson CW. Evaluation of benign vs malignant hepatic lesions with positron emission tomography. Arch Surg 1998;133(5):510-5; discussion 515-6.

24. Valk PE, Bailey DL, Townsend DW, Maisey MN. Positron Emission Tomography: Basic Science and Clinical Practice. London, Berlin, Heidelberg: Springer, 2003.
25. AJCC. AJCC Cancer Staging Manual. 6 th. Edition ed. Stuttgart, Heidelberg, New York: Springer; 2002.

26. Daisne JF, Duprez T, Weynand B, Lonneux M, Hamoir M, Reychler $\mathrm{H}$, et al. Tumor volume in pharyngolaryngeal squamous cell carcinoma: comparison at CT, MR imaging, and FDG PET and validation with surgical specimen. Radiology 2004;233(1): 93-100.

27. Tango T. Equivalence test and confidence interval for the difference in proportions for the paired-sample design. Stat Med 1998;17(8):891-908.

28. Adams S, Baum RP, Stuckensen T, Bitter K, Hor G. Prospective comparison of 18F-FDG PET with conventional imaging modalities (CT, MRI, US) in lymph node staging of head and neck cancer. Eur J Nucl Med 1998;25(9):1255-60.

29. Schoder H, Yeung HW. Positron emission imaging of head and neck cancer, including thyroid carcinoma. Semin Nucl Med 2004;34(3):180-97.

30. Anzai Y, Carroll WR, Quint DJ, Bradford CR, Minoshima S, Wolf GT, et al. Recurrence of head and neck cancer after surgery or irradiation: prospective comparison of 2-deoxy-2-[F-18]fluoro-D-glucose PET and MR imaging diagnoses. Radiology 1996;200(1):135-41.

31. Goerres GW, Schmid DT, Schuknecht B, Eyrich GK. Bone invasion in patients with oral cavity cancer: comparison of conventional CT with PET/CT and SPECT/CT. Radiology 2005;237(1):281-7.

32. Goerres GW, Von Schulthess GK, Hany TF. Positron emission tomography and PET CT of the head and neck: FDG uptake in normal anatomy, in benign lesions, and in changes resulting from treatment. AJR Am J Roentgenol 2002;179(5):1337-43.

33. Nakamoto Y, Tatsumi M, Hammoud D, Cohade C, Osman MM, Wahl RL. Normal FDG distribution patterns in the head and neck: PET/CT evaluation. Radiology 2005;234(3):879-85.

34. Steinkamp HJ, Hosten N, Richter C, Schedel H, Felix R. Enlarged cervical lymph nodes at helical CT. Radiology 1994;191(3):795-8.

35. Steinkamp HJ, Keske U, Schedel J, Hosten N, Felix R. The spiral CT of cervical lymph node enlargements. The initial clinical results. Rofo 1994;160(6):500-5.

36. Connell A, Corry J, Milner AD, Hogg A, Hicks RJ, Rischin D, et al. Clinical impact of, and prognostic stratification by, F-18 FDG PET/CT in head and neck mucosal squamous cell carcinoma. Head Neck 2007.

37. Wong WL, Hussain K, Chevretton E, Hawkes DJ, Baddeley H, Maisey M, et al. Validation and clinical application of computercombined computed tomography and positron emission tomography with 2-[18F]fluoro-2-deoxy-D-glucose head and neck images. Am J Surg 1996;172(6):628-32.

38. Sanghera B, Wong WL, Lodge MA, Hain S, Stott D, Lowe J, et al. Potential novel application of dual time point SUV measurements as a predictor of survival in head and neck cancer. Nucl Med Commun 2005;26(10):861-7.

39. Gutzeit A, Antoch G, Kuhl H, Egelhof T, Fischer M, Hauth E, et al. Unknown primary tumors: detection with dual-modality PET/ CT-initial experience. Radiology 2005;234(1):227-34. 